\title{
Implementing in situ Experiments in Liquids in the (Scanning) Transmission Electron Microscope ((S)TEM) and Dynamic TEM (DTEM)
}

\author{
Patricia Abellan ${ }^{1}$, Taylor J. Woehl ${ }^{2}$, Russell G. Tonkyn ${ }^{1}$, W. Andreas Schroeder ${ }^{3}$, James E. Evans ${ }^{4}$, Nigel \\ D. Browning ${ }^{1}$
}

${ }^{1}$ Fundamental and Computational Sciences Directorate, Pacific Northwest National Laboratory, P.O.Box 999, Richland, WA 99352, USA

2. Division of Materials Science and Engineering, U.S. DOE Ames Laboratory, Ames, IA 50011, USA

${ }^{3}$ Department of Physics, University of Illinois at Chicago, Chicago, IL 60607, USA

${ }^{4}$ Environmental Molecular Sciences Laboratory, Pacific Northwest National Laboratory, P.O. Box 999,Richland, WA 99352, USA

Developing a fundamental understanding of phenomena that take place in liquids, such as nanoparticle growth, self-assembly, protein conformational dynamics or the transformation of active materials during battery operation requires characterization tools able to provide in situ information with nanometer spatial resolution. In principle, this can be achieved using fluid stages in the (scanning) transmission electron microscope ((S)TEM). One of the main experimental challenges in the field of in situ liquid (S)TEM is obtaining reproducible data free of artifacts and beam-induced effects to enable quantitative analysis. Towards this aim, methods of calibration of the amount of radiation damage resulting from beam-induced reactions with the sample continue to be needed [1]. This magnitude strongly depends on the electron dose delivered to the sample [2-4]. For instance, in situ growth of particles in solution by the electron beam is typically observed in (S)TEM experiments and has been used to calibrate the effect of dose in a Ag precursor solution in an in situ fluid stage [3] (Figure 1 (a) shows areas where Ag growth experiments for different magnification were done). Custom image analysis algorithms using standard thresholding methods can be used to analyze movies of nanocrystal nucleation and growth and extract important information on growth dynamics and parameters such as the induction threshold below which no nucleation occurs [3] (see an example of image analysis in Figure 1(b)). Besides electron dose, factors such as accelerating voltage, imaging mode (e.g. TEM, STEM, SEM), liquid thickness, and solution composition are expected to affect the results of in situ experiments. Reproducing the result in a different instrument operating with different electron optical settings, introduces a large set of variables whose effect must be calibrated.

Fluid stages are specifically designed to fit in any transmission electron microscopy and thus, the different capabilities of each instrument can be applied to the study of liquid phase reactions. A combined temporal and spatial resolution of $\sim 10^{-6}$ and $\sim 10^{-10} \mathrm{~m}$, can be achieved by using fluid stages in combination with the dynamic TEM (DTEM) (see schematic of the DTEM design at PNNL in Fig. 1(c)). This spatio-temporal resolution covers a blind spot of current characterization techniques in catalysis, electrochemistry and biochemistry. Other advantages of the DTEM for the study of nanomaterials dynamics in liquids include the ability of initiating a reaction independently from the electron beam [4] and quantified dynamics by removing the effect of particle motion in solution. To achieve the resolution described above, the Dynamic TEM (DTEM) laser system at the Pacific Northwest National Laboratory (PNNL) is specified for single-shot imaging operation generating $>10^{8}$ electrons per pulse - this implies a UV laser pulse energy incident on the photocathode of greater than $0.1 \mathrm{~mJ}$. The initial pulse duration (laser and hence also electron) will be 1 microsecond (although shorter pulse durations are possible). The DTEM itself uses a dual aberration corrected JEM 2200FS base microscope and two lasers- the UV photocathode pulse at 
$266 \mathrm{~nm}(4.67 \mathrm{eV})$ and the specimen perturbation pulse at $1064 \mathrm{~nm}, 532 \mathrm{~nm}$ or $355 \mathrm{~nm}$. Cs-correction and a phase plate are being developed for improved contrast and resolution.

Here, we present our recent developments in the design and implementation of calibration experiments using in situ fluid stages, including an identification of beam-sample interactions for changing imaging and experimental conditions. The application of these stages to studying the dynamics of nanomaterials in liquids using the DTEM will also be discussed.

References:

[1] T.J. Woehl et al., Ultramicroscopy 127 (2013) 2927; J.M. Grogan, Nano Letters 14 (2014) 359 [2] H.M. Zheng et al., Nano Letters 9 (2009) 2460

[3] T.J. Woehl et al., ACS Nano 6 (2012), p. 8599; T.J. Woehl et al., Nano Letters 14 (2014), p. 373.

[4] J.E. Evans et al., Nano Letters 11 (2011) p. 2809

[5] J.E. Evans et al., Microscopy 62 (2013) p 147-156

[6] This work was supported by the Chemical Imaging Initiative; under the Laboratory Directed Research and Development Program at Pacific Northwest National Laboratory (PNNL). PNNL is a multiprogram national laboratory operated by Battelle for the U.S. Department of Energy (DOE) under Contract DE-AC05-76RL01830. A portion of the research was performed using the Environmental Molecular Sciences Laboratory (EMSL), a national scientific user facility sponsored by the Department of Energy's Office of Biological and Environmental Research and located at Pacific Northwest National Laboratory.
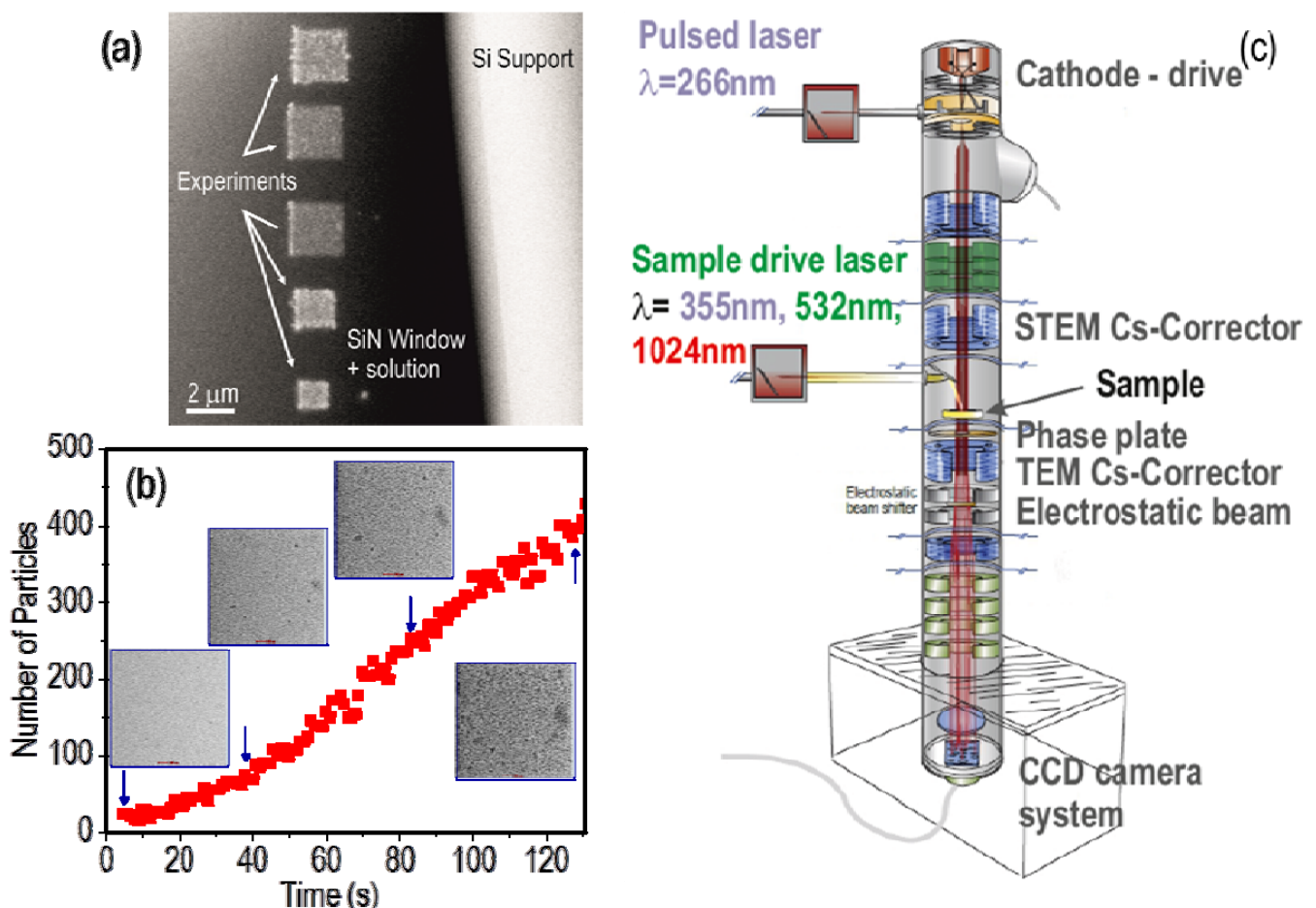

Figure 1.(a) BF STEM image showing a set of experiments in the fluid stage where Ag nanocrystals were grown from solution using the electron-beam for different magnifications. (b) Number of particles grown as a function of time measured from an in situ dataset using $300 \mathrm{kV}, 7.1 \mathrm{pA}$ beam current, $3 \mu$ s pixel-dwell time and $M=40000 x$ in STEM, to give a dose per frame of $39.1 \mathrm{e}-/ \mathrm{nm}^{2} \mathrm{f}$. (c) Schematic of the DTEM showing the upgrades planed at PNNL. Modified from [5]. Copyright 2013 Oxford University Press. 